\title{
Thermomechanical design in the automotive industry
}

\author{
J. J. THOMAS, L. VERGER, A. BIGNONNET and E. CHARKALUK \\ PSA Peugeot Citro"enm, Applied Mechanics Research Division, Route de Gisy, 78943, V'elizy Villacoublay Cedex, France
}

\begin{abstract}
A B S TRA C T In this paper thermomechanical fatigue assessment in the automotive industry is discussed. The design strategy is based upon a consistent approach of the thermomechanical loading, the mechanical constitutive law of the material, the damage parameters and the fatigue strength criteria. The good understanding of these different steps allows one to perform predictive calculations of automotive parts subjected to thermomechanical loading. The main hypotheses and modelling choices are presented and results are illustrated by a series of computations on real 3D structures. Cracked area and lifetime prediction are described in the case of aluminium alloy cylinder heads subjected to transient thermal loadings.
\end{abstract}

Keywords automotive; constitutive model; engine; fatigue; thermomechanical; viscoplastic.

\section{INTRODUCTION}

Development in numerical simulation over the past 20 years have led to increased performance of numerical tools and the development of new algorithms. Today this enables ever-quicker calculations of loads and displacements in structures. Nevertheless, whilst academic work is quite active few studies have been performed on structures subjected to multiaxial thermomechanical loading.

Only a small number of components in automotive technology experience thermomechanical loading cycles of a severity capable of producing low-cycle fatigue. Most of these parts are related to the engine: cylinderheads, exhaust manifolds, crankcases, .... It is important to note that the low-cycle fatigue problems in such engine components are related to the start-operate-stop cycles and not to the combustion cycles. Therefore they involve large temperature changes. A previous study was dedicated to cast-iron exhaust manifolds. ${ }^{1}$ This work showed an original comprehensive approach, which resulted in a reliable prediction of damage location and of lifetime in spite of basic assumptions like the separation of the damage and the mechanical constitutive behaviour.

The paper presents an overview of this approach and the specific details of the applications in the case of the cylinder head in aluminium. The results are illustrated by a series of computations on real 3D structures. The words comprehensive approach shall denote the following series of items:

Correspondence: Jean-Jacques Thomas. E-mail: jeanjacques.thomas@mpsa.com
- thermal analysis,

- elasto-viscoplastic mechanical analysis and

- fatigue analysis.

These three aspects are linked together; the thermal loading and the material behaviour determine the mechanical response of the structure and from that the fatigue strength of the structure can be estimated through a damage indicator and a pertinent fatigue criterion.

For a design strategy, one should not concentrate separately on these four aspects of the problem. It should be a comprehensive approach to obtain an important robustness and to allow its integration in a development process ${ }^{2}$ by the ability to answer the asked question in the appropriate time. That is to say in our case: is the fatigue strength of the component guaranteed or not before the production of tooling for a prototype?

The cylinder head is made of an aluminium alloy (in this case an A356 alloy) and locally experiences temperature cycles ranging from 20 to $300^{\circ} \mathrm{C}$. The mechanical behaviour of the aluminium alloy is plastic at low temperatures and highly viscoplastic at the upper temperatures. Moreover, aluminium alloys are also subjected to thermal ageing effects for temperatures over $150^{\circ} \mathrm{C}$.

Therefore different steps had to be defined in order to apply the comprehensive approach in this case:

- modelling of the ageing behavior of the material,

- modelling of the cyclic viscoplastic behavior of the material,

- calibration of the parameters of the models on the basis of isothermal and non-isothermal uniaxial tests, 

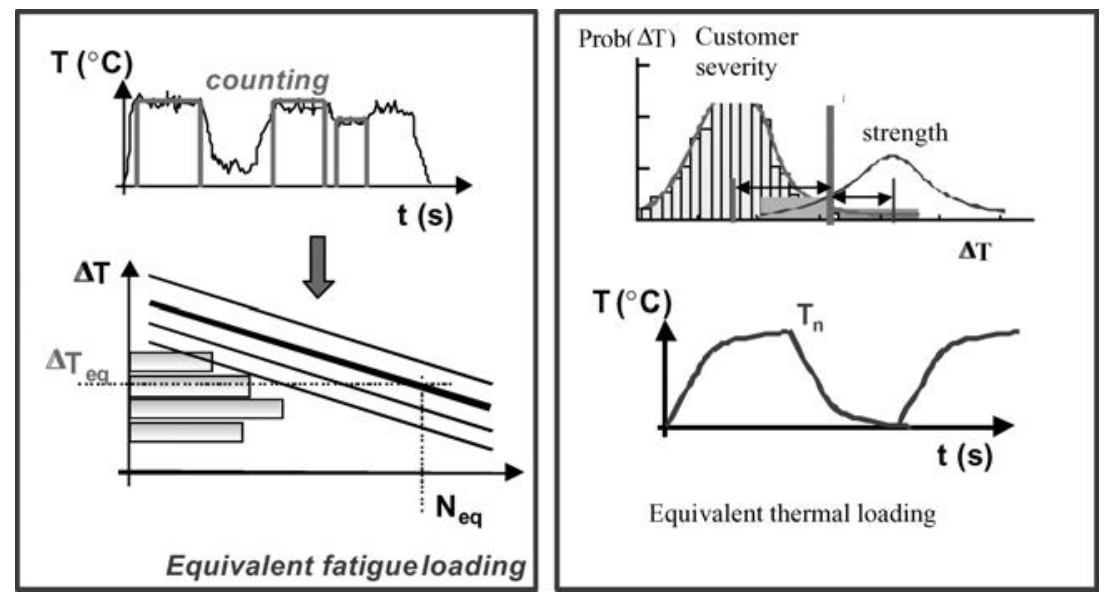

Fig. 1 Transformation of in service temperature analysis to an equivalent loading, with a known severity for tests or calculation, and decision strategy.
- determination of a low-cycle fatigue criterion on the basis of isothermal uniaxial tests and non-isothermal uniaxial and multiaxial tests,

- computation of the finite-element analysis with realistic boundary and loading conditions and

- determination of the lifetime of the structure using the chosen fatigue criterion.

In order to keep the above methodology at a complexity level compatible with the constraints of the industrial design engineers, several assumptions regarding the $u n$ coupling of the different phenomena have been made: uncoupling between thermal computation and mechanical computation; uncoupling between ageing evolution and mechanical behaviour and uncoupling between the constitutive law and the damage modelling. Another important hypothesis is the existence of a stabilized mechanical response of the structure. The design strategy is based on the assumption that an acceptable component will rapidly reach a stabilized response to the cycling loading. It means that shakedown will occur. Rather than simulating the whole life of a component, it is then possible to focus on the stabilized response of the structure and to extract some damage indicator from this cycle. To save time, one must define an efficient method to rapidly reach this stabilized response. One must also define a relevant damage indicator which will permit prediction of the lifetime.

One of the main difficulties exists in the fact that the calibration of the temperature dependant parameters of the constitutive law with non-isothermal tests data could not be simplified. ${ }^{3}$

\section{REPRESENTATION OF INSERVICE LOADING}

In the first step it is important that the thermal loading applied at the validation stage on engine testing and while calculating virtual prototype should be representative of the customer's uses from the point of view of the fatigue damage. An analysis method, which can be used, is the stress-strength interference analysis ${ }^{4}$ that is frequently used for high-cycle fatigue studies.

The main elements of this method are: a counting method (e.g., rain-flow counting) on the appropriate and accessible variable (e.g., forces, accelerations and temperatures); a cumulative damage law or equivalent damage method (e.g., Miner's rule) and a risk analysis and the description of the fabrication scatter (material and process).

The extension of this method to the thermomechanical fatigue is particularly delicate due to the non-linearity (behaviour, loading, ...) and assumes several simplifying hypothesis. Nevertheless, this can be reasonably done and is used to define bench test to represent the customer's use with the desired severity. Figure 1 schematically represents the different steps of this work for a typical thermal loading of an exhaust pipe. The acceptance criterion is given with regard to an objective customer taking into account the fabrication scatter on a definite risk.

\section{SIMULATION OF THE THERMAL LOADING}

For engine components, the main element of the loading is the thermal evolution of the components in operating engine conditions defined to be representative of customer use (the mechanical loading is essentially linked to the bolt tightening between the different components and to the press fit of valve seats or valve guides in the case of cylinder head; these different aspects can be represented rather easily). This is a dominant step of the approach, because the accuracy of thermal data conditions governs the accuracy of the mechanical response of the structure and, therefore, the estimated fatigue strength.

The input data come from engine combustion simulation using numerical tools (KIVA, FIRE, FLUENT, ...). In the case of the exhaust manifold, these data are associated with the gas flow in the exhaust ports and pipes to determine the gas temperatures, and therefore, to 
determine the internal exchange coefficient. For the cylinder head, they are used as thermal flux on the fire deck. A transient thermal calculation done in both cases enables the determination of the thermal loading hich is used for the thermomechanical calculation. ${ }^{5}$

The determination of the thermal loading is still the major difficulty of a thermomechanical fatigue approach in the automotive industry (particularly for thermal exchange between the engine components: cylinder head, crankcase, piston, exhaust pipe,...) and will necessitate extensive work in the forthcoming years.

\section{MODELLING THE MATERIAL BEHAVIOUR}

The origins of failures encountered at high temperature in automotive components is linked to thermal loading. Three main aspects can be underlined:

- The temperature cycles between ambient and maximum temperature $\left(250^{\circ} \mathrm{C}\right.$ in the cylinder head, $800^{\circ} \mathrm{C}$ and above on exhaust pipe or $700{ }^{\circ} \mathrm{C}$ for brake disk) reach a domain where mechanical strength of material becomes quite low.

- High temperature dwell time enhances creep (that is the case for engine crankcases).

- Steep thermal gradients leads to inelastic deformations in confined zones.

Failure analysis also evidences the cyclic aspects of the loading with high and variable temperatures.

Within these temperature ranges $\left(0.3-0.5 T_{\mathrm{m}}\right.$ - melting point) the behaviour of the material is strongly non-linear. We can no longer speak of elasticity.

Material testing is performed on material/process representative specimens (similar microstructure and metallurgical quality as the actual component) at different strain rates with dwell times at imposed strains. The material behaviour shows a strain rate dependence of this threshold and a stress recovery during dwell time. As an example, the mechanical behaviour of a $356 \mathrm{Al}$ alloy is given in Fig. 2 .

Experimentations at constant stress reveal creep of the material. These phenomena clearly demonstrate the role of viscosity and therefore a simple plasticity model is not able to represent our problem. We are faced with an elastoviscoplastic behaviour.

For the modelling of high temperature deformation, mechanisms have been described by several authors (François et al., ${ }^{6}$ Lemaitre and Chaboche ${ }^{7}$ ). Most of the work on that field started with the growth of the nuclear industry and the aeronautics. Two approaches have been particularly developed at this time: the unified theory characterized by constitutive law with a unique inelastic $\operatorname{strain}^{8,9}$ and the damage mechanics initiated by Kachanov ${ }^{10}$ and further developed by Chaboche. ${ }^{11}$ If these results are often used, in aeronautics for example, they present some disadvantages:

- The constitutive law can rapidly become complex to model all the physical phenomena. The number of characteristic parameters becomes quite important and their eventual coupling makes their calibration difficult.

- These mechanical models are not necessarily representative of the whole physical phenomena, because they are often imperfect to model both creep and stress relaxation (François et al. ${ }^{6}$ ).

- Coupling damage and constitutive behaviour lead to difficulties in parameters calibration and numerical implementation, and overall leads to lengthy calculation.

Based on these observations, one can conclude that to be appropriate to the automotive context, thermomechanical modelling is subjected to several constraints:

- The necessity is to represent the behaviour of the material in the structure by simple constitutive equations. In this
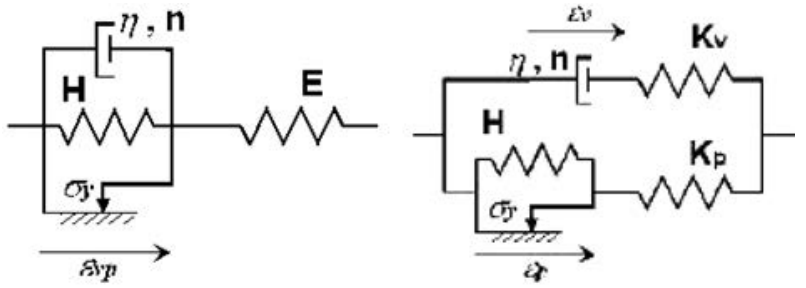

Fig. 3 Unified model and viscoelastic-elastoplastic model.
Fig. 2 Influence of the temperature and evidence of the stress relaxation for a $356 \mathrm{Al}$ alloy.
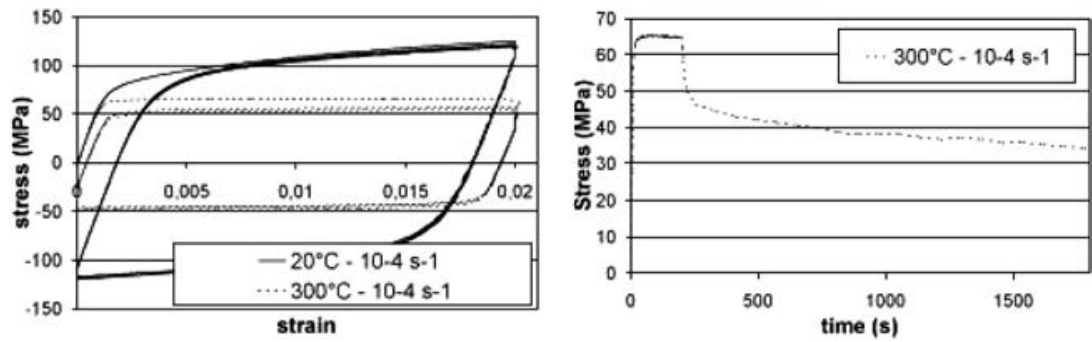
context, one should adopt the representation of the mechanical behaviour without coupling with damage (Fig. 3). From experience, most of the times this coupling is not necessary and does not improve the predictivity of the behaviour of the structure. ${ }^{13}$ Usually it is not necessary to describe the evolution of the structure cycle by cycle and particularly the early stage of the life of the structure. Therefore the calibration of the parameters of the constitutive equation can be done on steady-state cycles from material testing.

- The implementation in a finite-element code using a numerical integration algorithm for the constitutive law stable and robust enough to enable large integration steps.

The unified model can be described by the following equations:

$\varepsilon=\varepsilon^{e}+\varepsilon_{\mathrm{vp}}, \boldsymbol{\sigma}=\mathbf{C}: \varepsilon^{e}, \boldsymbol{\alpha}=\frac{3}{2} \frac{1}{H} \mathbf{X}$,

$\dot{\varepsilon}_{\mathrm{vp}}=\frac{3}{2}\left\langle\frac{\mathcal{F}_{2}(\boldsymbol{\sigma}-\mathbf{X})-\sigma_{\mathrm{y}}}{\eta}\right\rangle^{m} \frac{\mathbf{s}-\mathbf{X}}{\mathcal{F}_{2}(\mathbf{s}-\mathbf{X})}=\dot{\boldsymbol{\alpha}}$,

where $\langle x\rangle$ denotes the positive part of $x, \mathcal{F}_{2}(\mathbf{a})=$ $\sqrt{\frac{3}{2} \operatorname{dev}(\mathbf{a}): \operatorname{dev}(\mathbf{a})}, \mathbf{s}=\operatorname{dev}(\boldsymbol{\sigma})$ and $\mathbf{X}$ is the hardening.

The viscoelastic-elastoplastic model can be described by the following equations:

$\varepsilon=\varepsilon_{\mathrm{v}}^{\text {elas }}+\varepsilon_{\mathrm{v}}, \quad \varepsilon=\varepsilon_{\mathrm{p}}^{\mathrm{elas}}+\varepsilon_{\mathrm{p}}, \quad \alpha=\frac{3}{2} \frac{1}{H} \mathbf{X}$,

$\varepsilon_{\mathrm{v}}^{\text {elas }}=\frac{1+v}{K_{\mathrm{v}}} \boldsymbol{\sigma}_{\mathrm{v}}-\frac{v}{K_{\mathrm{v}}} \operatorname{tr}\left(\boldsymbol{\sigma}_{\mathrm{v}}\right) \mathbf{I}, \quad \varepsilon_{\mathrm{p}}^{\mathrm{elas}}=\frac{1+v}{K_{\mathrm{p}}}$

$\boldsymbol{\sigma}_{\mathrm{p}}-\frac{v}{K_{\mathrm{p}}} \operatorname{tr}\left(\boldsymbol{\sigma}_{\mathrm{p}}\right) \mathbf{I}$

$\dot{\varepsilon}_{\mathbf{v}}=\frac{3}{2}\left\langle\frac{\mathcal{f}_{2}\left(\boldsymbol{\sigma}_{\mathrm{v}}\right)}{\eta}\right\rangle^{m} \frac{\mathbf{s}_{\mathrm{v}}}{\mathcal{f}_{2}\left(\boldsymbol{\sigma}_{v}\right)}, \quad \dot{\varepsilon}_{\mathrm{p}}=\gamma \frac{\mathbf{s}_{\mathrm{p}}-\mathbf{X}}{\mathcal{f}_{2}\left(\boldsymbol{\sigma}_{\mathrm{p}}-\mathbf{X}\right)}=\dot{\boldsymbol{\alpha}}$.

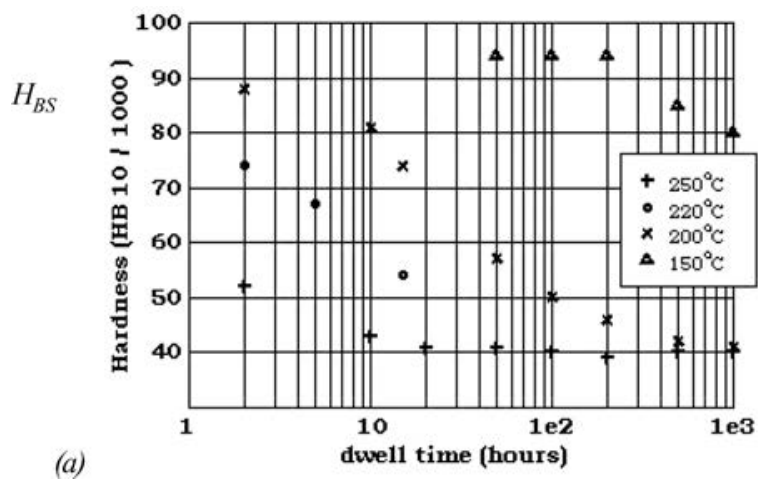

\section{Cylinder head}

The studied automotive cylinder heads are made of an A356 aluminium alloy composed of $7 \%$ silicon and $0.3 \%$ magnesium (in mass percentage). In critical areas during the engine processing, the temperature of the material can reach the value of $300^{\circ} \mathrm{C}$.

At these temperatures, the material shows two main behaviours: on one hand a considerable thermal ageing resulting in the loss of at most $70 \%$ of its mechanical characteristics, on the other hand a highly viscoplastic mechanical behaviour as the temperature approaches $60 \%$ of the melting temperature.

\section{Ageing behaviour}

In order to optimize the mechanical characteristics of the studied alloy at an upper level despite of the high temperature, the entire cylinder head is submitted to a typical T7 thermal treatment after casting: homogenization at $540{ }^{\circ} \mathrm{C}$ to dissolve the precipitates, water quenching, artificial ageing at $200{ }^{\circ} \mathrm{C}$ during which the components $\mathrm{Mg}_{2} \mathrm{Si}$ precipitates are formed.

The temperature influence on this original $\mathrm{T} 7$ alloy has been measured during an appropriate experimental programme of conventional yield stress and hardness measurements on specimens submitted to constant temperature dwells (Fig. 4).

A quite simple exponential function has been used to model the temperature and time-dependant ageing of the alloy:

$$
\begin{aligned}
& H_{\mathrm{B}}(t, \theta)= \\
& H_{\mathrm{BS}}+\frac{\left(H_{\mathrm{B} 0}-H_{\mathrm{BS}}\right)-\exp (0.95 \ln (\theta)+15.7 \ln (t)-86.4)}{1+\exp (0.95 \ln (\theta)+15.7 \ln (t)-86.4)},
\end{aligned}
$$

with $H_{\mathrm{B} 0}$ the original hardness in Brinell scale, and $H_{\mathrm{BS}}$ the hardness of the fully aged material.

Fig. 4 Temperature ageing evaluation of a T7 A356 aluminium alloy ${ }^{12}$ by the evolution of the hardness (a) and conventional yield stress (b). 
This function has proved to be correctly fitting the experimental data on specimens ${ }^{12}$ as well as on processed cylinder heads (Fig. 5).

\section{Mechanical behaviour}

Several tests have been performed on specimens in order to show the cyclic mechanical behaviour of A356 aluminium alloy (Fig. 6). These are tension/relaxation/compression (TRC) cyclic tests at different temperatures in the range $20-300{ }^{\circ} \mathrm{C}$, performed at various strain rates: $10^{-3} \mathrm{~s}^{-1}, 5.10^{-4} \mathrm{~s}^{-1}$ and $10^{-4} \mathrm{~s}^{-1}$.

A viscous behaviour has been evidenced by exponential stress/time relaxation response. Plastic yield stress and hardening are noticed at each temperature. A classical viscoplastic model with one single inelastic strain variable similar to those developed by Chaboche $^{7}$ has then been used with temperature-dependant parameters (Fig. 3).

\section{Uncoupled damage}

As some classical low cycle fatigue (LCF) tests have been performed in the same range of temperatures, it has been shown that the A356 aluminium alloy undergoes a slight cyclic softening during almost the whole life of the specimens (Fig. 7). The coupled modelling of such a cyclic damage with the viscoplastic behaviour would be very expensive in terms of identification tests and computation times. Furthermore, several studies have already shown
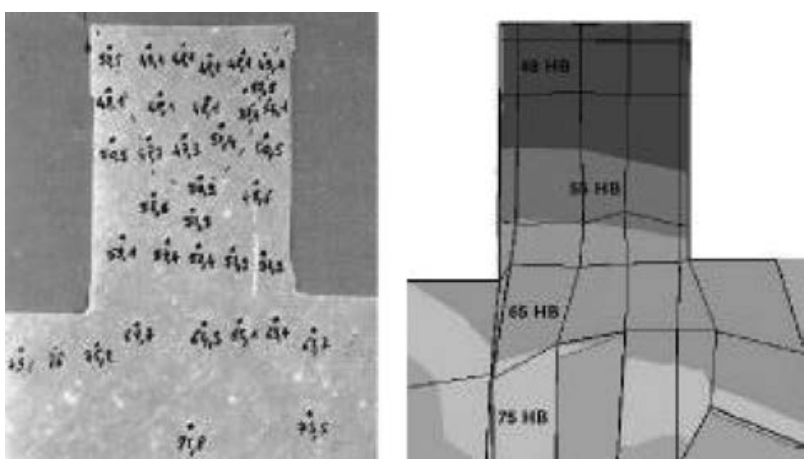

Fig. 5 Comparison of hardness measurements (a) and calculations (b) on a section of intervalve space of a tested cylinder head. that this coupling may not be necessary to perform reasonably predictive lifetime calculations. 1,13

As a result, it has been chosen not to include any damage variable in the constitutive law used to represent the mechanical response of the studied material. The cyclic damage will then be calculated from the mechanical variable results of a 'stabilized cycle' numerical simulation (for more details, see the fatigue design part of the present document).

\section{Constitutive law calibration}

The unified viscoplastic model chosen in this case is available over the whole range of temperature provided that its five parameters are computed as temperature-dependant functions.

The calibration method is based on an optimal control approach. ${ }^{14}$ The parameters as functions of temperature are determined through the minimization of a cost function defined as

$\mathcal{f}(p)=\frac{1}{2} \int\left[\sigma_{\exp }-\sigma_{\text {calc }}(p, t)\right]^{2} \mathrm{~d} t$,

where the time integral measures the difference between the experimental and the computed stresses $\left(\sigma_{\exp }\right.$ and

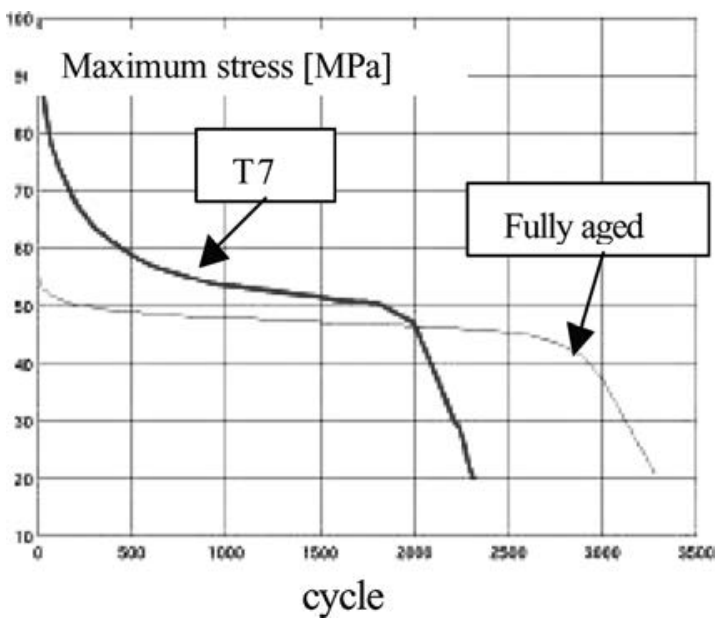

Fig. 7 Maximum stress during LCF strain controlled tests at constant temperature.
Fig. 6 Response of T7 (a) and fully aged (b) specimens to a cyclic TRC test at several temperatures.

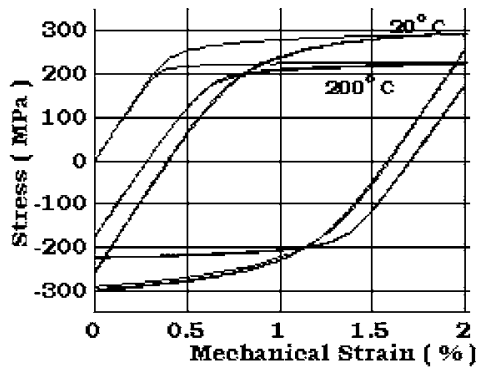

(a)

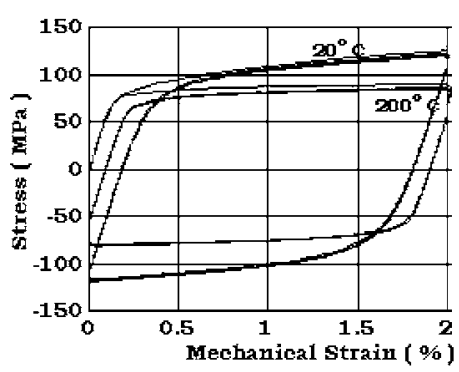

(b) 
$\sigma_{\text {calc }}$ ), respectively, over a complete stabilized cycle. In order to determine parameters from several experiments, the corresponding cost functions can be added using weight factors. The minimization itself is performed by a classical BFGS gradient descent algorithm.

According to the ageing of the alloy, both measured and calculated on the structure (Fig. 5), it has been chosen to calibrate the material parameters under two stable states: on one hand the original T7 state which makes up the 'cool' part of the cylinder head, and on the other hand the fully aged state found in the 'hot' part of the structure, which undergoes the thermomechanical fatigue phenomena. By means of a set of calculations on structure, the consequences of the lack of realistic ageing modelling of the second order in the transitional area on the fatigue predictions have been shown.

A particular effort had to be made on the determination of the experimental database to be used for the identification analysis. ${ }^{3}$ Indeed the viscosity parameters $\eta$ and $m$ show an important coupling behaviour on the TRC tests simulation at constant temperature (Fig. 8). Then, the use of non-isothermal experimental data provide us with a useful additional information. Furthermore, the loading conditions applied on specimens for non-isothermal tests

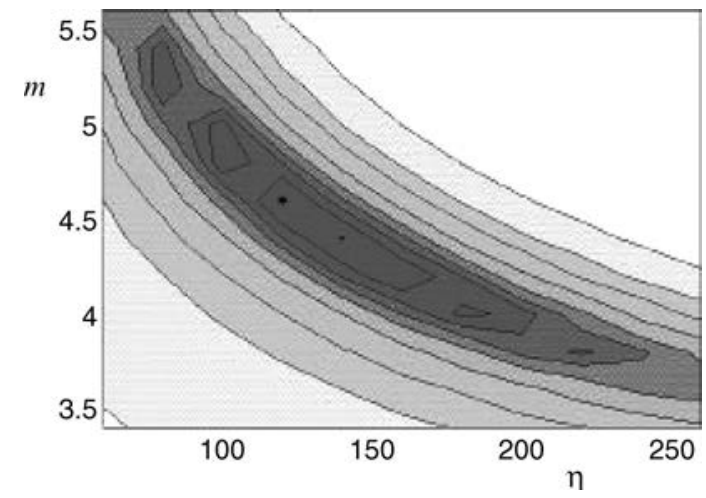

Fig. 8 Isovalue of the cost functional showing the $(\eta, \mathrm{m})$ coupling in the length of its minimum valley. can be defined using the typical thermal and strain history recorded on the engine component (Fig. 9). As a result, the parameters identified from both isothermal TRC and non-isothermal 'structure-like' measurements are likely to perform realistic behaviour simulations on structure calculations.

\section{FATIGUE ANALYSIS}

The final objective is to evaluate the fatigue strength, or the lifetime of the structure, from the stabilized response of this structure. A criterion must then be defined upon the mechanical variables linked to the stress and strain fields. The first studies on the LCF criteria give some useful parametric laws. Nevertheless, all the classical approaches like Manson-Coffin, ${ }^{15,16}$ strain range partitioning ${ }^{17}$ (SRP) or the function defined by Smith-Watson-Topper ${ }^{18}$ (SWT) initially expressed from uniaxial isothermal tests were not really adaptable to the thermomechanical fatigue of the complex structures. The difficulties came from the multiaxial character of the loading of such structures as well as the large temperature range seen under operation. Actually the usual interpretation of low cycle isothermal fatigue tests with the Manson-Coffin law shows a large differences in fatigue life for test with the same strain amplitude but at different temperature (as shown on Fig. 10 for cast iron), and it is not clear how it can be generalized to the multiaxial case. Furthermore, the predictions by means of cumulative plastic strain as proposed by the strain range partitioning method on a stress-strain isothermal curve is somewhat hazardous.

An analysis of an anisothermal test using the SWT function (SWT $=\sqrt{E \sigma_{\max } \Delta \varepsilon}$ ) is difficult because the choice or the definition of $\sigma_{\max }$ had no real sense and is in fact impossible in the anisothermal case. Therefore the major criticism for all these criteria is the impossibility of their generalization to complex loadings.

This situation is also the case for the creep fatigue laws developed by Chaboche. ${ }^{19}$ These laws have been generalized to anisothermal cases using a reduced stress $\sigma / \sigma_{u}$,

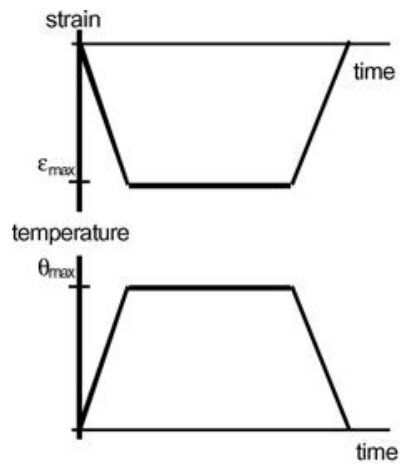

(a)

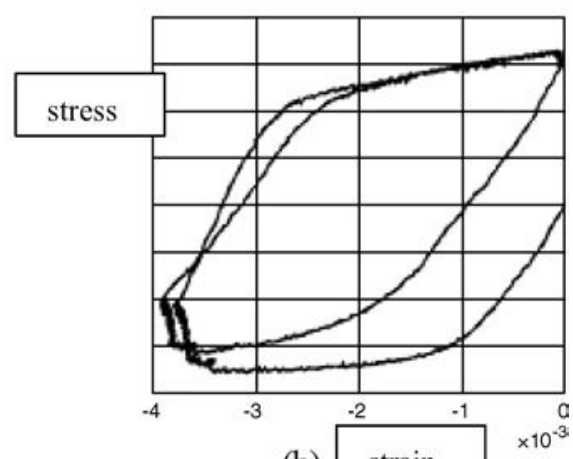

(b)
Fig. 9 Strain and temperature conditions of the non-isothermal test (a) and the stress/strain response of the viscoplastic model (b). 
where $\sigma_{\mathrm{u}}$ is the ultimate strength at the considered temperature, used to define the maximal stress or the mean stress independently of the temperature. This reduced variable is not justified from mechanical and physical point of view. For that reason Chaboche ${ }^{19}$ proposed to redefine the damage law in the anisothermal context.

A possible way to overcome these difficulties is the energetic approach. Skelton ${ }^{20}$ describes the damaging process by the growth of crack through a process zone of constant size $\rho$ at the crack tip. He considered the growth of the crack when a sufficient quantity of energy has been cumulated in this process zone. From that Charkaluk and Constantinescu ${ }^{1}$ observed that the dissipated energy per cycle, at a steady-state $\Delta W_{\mathrm{S}}$, can be representative of the fatigue behaviour of a material:

$$
\Delta W_{\mathrm{S}}=\int_{t}^{t+T} \sigma \mathrm{d} \varepsilon_{\mathrm{p}}
$$

The advantage of the dissipated energy per cycle comes from three main points:

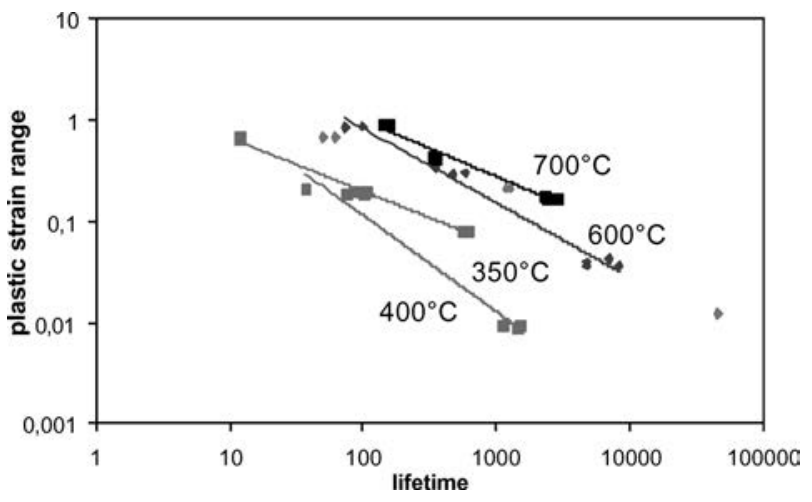

Fig. 10 Manson Coffin curve for cast iron at 200, 350, 400, 600 and $700{ }^{\circ} \mathrm{C}$.

(a)
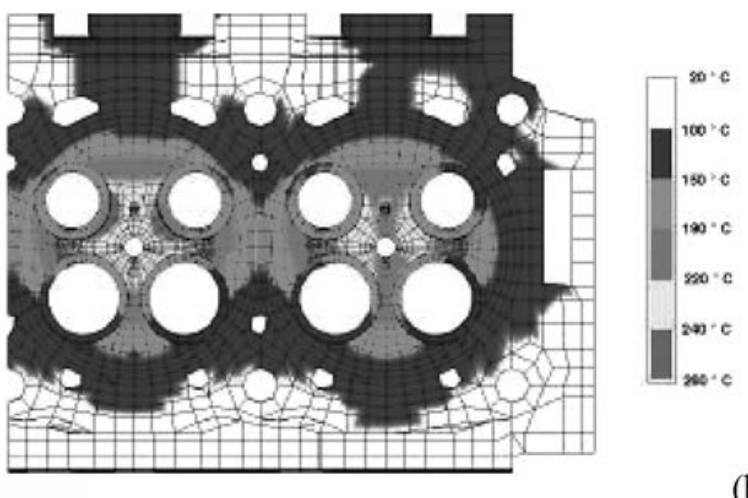

(b)

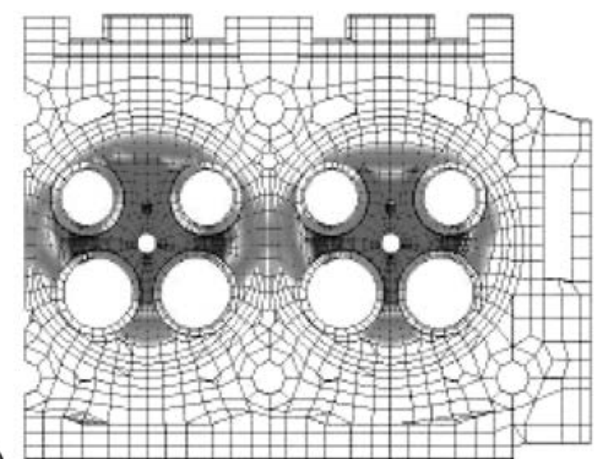

Fig. 11 Maximum temperature field (a) and hardness distribution after 10000 cycles (b) computed on a cylinder head subjected to thermomechanical cycles. 
Numerical simulation and fatigue strength prediction

For such complex models, the submodelling technique is recommended. It consists of a global elastic calculation of the structure. For the area where the behaviour is strongly non-linear a sub model is extracted. The displacements at the boundary of the submodel and the temperature are then applied to this submodel to perform the viscoplastic calculation.

This technique allows to limit the viscoplastic calculation only where it is necessary. This helps to minimize the calculation time, which can be typically $2 \times 10^{5} \mathrm{~s}$ CPU.

Once the mechanical fields of a stabilized cycle are obtained, the fatigue analysis consists of applying the dissipated energy LCF criterion.

\section{Fatigue assessment}

In the case of the A356 alloy, the fatigue criterion has been established using classical isothermal LCF tests on fully artificially aged specimens at different temperatures between 20 and $300{ }^{\circ} \mathrm{C}$ under strain control. A series of TMF (thermo mechanical fatigue) tests on clamped specimens were also conducted to assess the fatigue criterion.

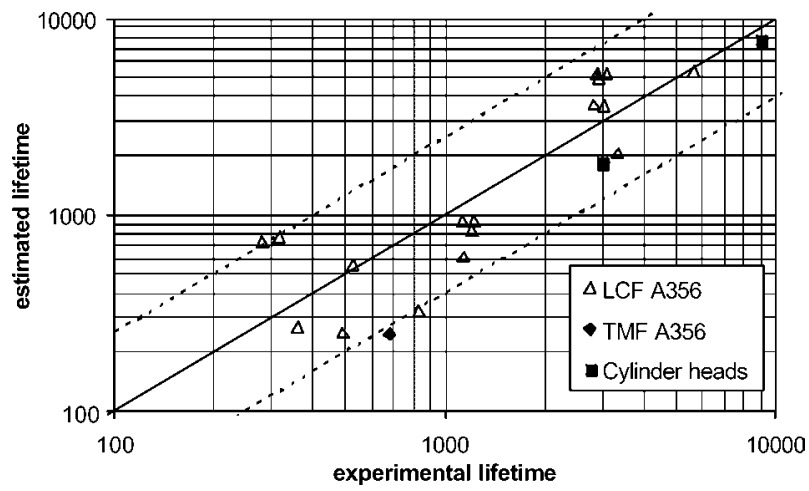

Fig. 12 Comparison of the estimate lifetime (in cycles) and experimental lifetime for A356 alloy LCF and TMF tests and several structures.
Figure 12 represents the comparison between experimental lifetime and calculated results for these different tests, as well as for two real structure calculations. All the lifetime results stay within an acceptable \pm 2.5 error margin on lifetimes.

The prediction of the crack location (Fig. 13) as well as the lifetime has been proved to be fully satisfactory on complete structure calculations. It should be noticed that these results have been achieved on large FEM models of more than 800000 degrees of freedom in computing times compatible with the constraints of the industrial design engineers.

\section{CONCLUSION}

A complete design approach has been proposed for structures undergoing thermomechanical fatigue. Formerly applied to cast iron exhaust manifolds, the method is developed here for the design of aluminium alloy cylinder heads. Based on the use of a simple elasto-viscoplastic law and an energetic failure criterion both suitable under multiaxial and non-isothermal loading conditions, the design approach also calls for some important assumptions regarding the uncoupling of modelled phenomena which permit the computations of large structures. This design approach has been successfully applied on several industrial structures. It has shown a good correlation between prediction and experiment for the crack initiation location as well as for the durability, which demonstrates the robustness of the approach. Moreover this method is compatible with an engine development schedule and permits to decrease the number of validating thermal shock tests on real components.

\section{REFERENCES}

1 Charkaluk, E. and Constantinescu, A. (2000) Energetic approach in thermomechanical fatigue for silicon molybdenum cast-iron. Mater. High Temp. 17, 373-380.

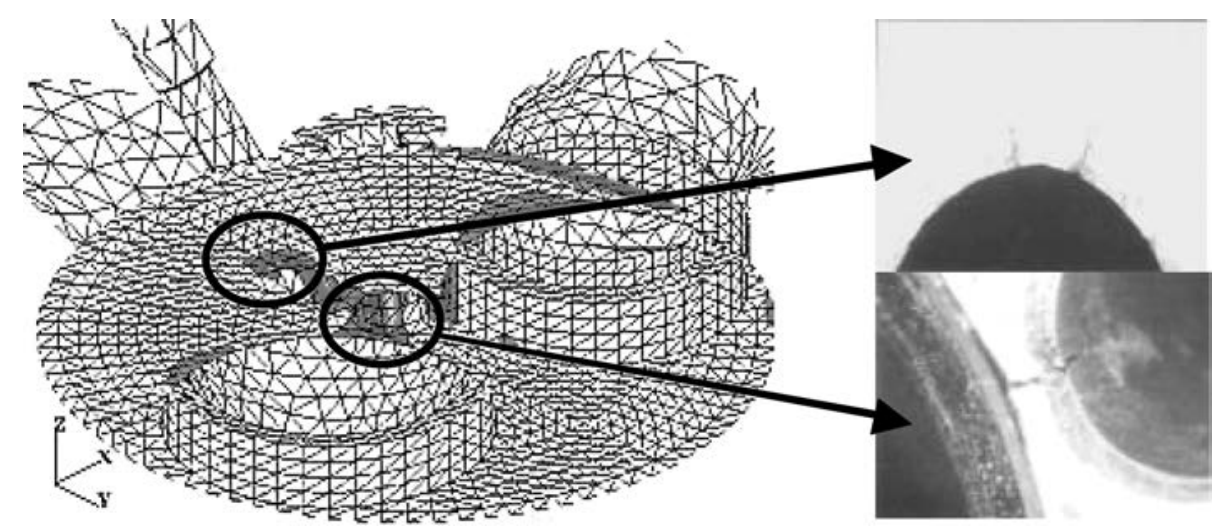

Fig. 13 Crack initiation on a cylinder head prototype and distribution of the fatigue criterion on the FE model. 
2 Charkaluk, E., Constantinescu, A., Bignonnet, A. and Dang Van, K. (1999) Dimensionnement en fatigue des structures, SF2M 18th Journées de printemps.

3 Verger, L., Constantinescu, A. and Charkaluk, E. (2000) IUTAM Creep in Structures 2000 (Edited by S. Murakami \& N. Ohno), Kluwer, North Holland.

4 Thomas, J. J., Perroud, G., Bignonnet, A. and Monnet, D. (1998) Fatigue design and reliability in the automotive industry. In: Proceedings of the Fatigue Design'98-3rd International Symposium on Fatigue Design (Edited by G. Marquis), pp. 1-11.

5 Lederer, G., Charkaluk, E., Verger, L. and Constantinescu, A. (2000) Numerical lifetime assessment of engine parts submitted to thermomechanical fatigue, application to exhaust manifold design. SAE Technical Paper Series, 2000-01-0789.

6 Francois, D., Pineau, A. and Zaoui, A. (1994) Comportement mécanique des matériaux, vol II, Hermès.

7 Lemaitre, J. and Chaboche, J. L. (1990) Mechanics of Solid Materials, Cambridge University Press, Cambridge, UK.

8 Sehitoglu, H., Qing, X., Smith, T., Mayer, H. J. and Allison, J. A. (2000) Stress strain response of a cast 319-T6 aluminium under thermomechanical loading. Met. Trans. A 31, 139-151.

9 Nicouleau-Bourles, E., El-Mayas, N., Massinon, D. and Cailletaud, G. (1999) Thermomechanical fatigue of aluminium alloys: experimental study and numerical simulation. In: Thermal Stresses 99, Third International Congress of Thermal Stresses, Cracovie, pp. 241-244.

10 Kachanov, L. M. (1958) Time on the rupture process under creep conditions. Izv. Akad. Nauk., S.S.R., Otd. Tekb. Nauk. 8, 26-31.

11 Chaboche, J. L. and Stoltz, C. (1974) Détermination des durées de vie des aubes de turbines à gaz. Revue Française de Mécanique 50-51, 71-82.

12 Boussac, O. and Callais, T. (1998) Influence du Vieillissement Thermique sur les Caractéristiques Mécaniques à Température Ambiante de l'AS7G03 Y39, Rapport Interne PSA.

13 Sermage, J. P., Lemaitre, J. and Desmorat, R. (2000) Multiaxial creep-fatigue under anisothermal conditions. Fatigue Fract. Engng Mater. Struct. 23, 241-252.

14 Bourgeois, L. (1998) Contrôle Optimal et Problèmes Inverses en Plasticité. PhD report, Ecole Polytechnique (Paris).

15 Coffin, L. F. (1953) A study of the effects of cyclic thermal stresses on a ductile material. Trans. ASME 53-A76, 931-950.

16 Manson, S. S. (1953) Behaviour of materials under conditions of thermal stresses. Technical Report TN 2933, NACA.

17 Halford, G. R. and Manson, S. S. (1976) Life prediction of thermal-mechanical fatigue using strainrange partitioning. In Thermal Fatigue of Materials and Components. ASTM STP 612.

18 Smith, K. N., Watson, P. and Topper, T. H. (1970) A stress-strain function for the fatigue of metals. 7. Mater. $\mathbf{5}$, 767-778.

19 Chaboche, J. L. (1974) Une loi différentielle d'endommagement de fatigue avec cumulation non linéaire. Revue Française de Mécanique 50-51, 71-82.

20 Skelton, R. P. (1991) Energy criteria for high temperature low cycle fatigue. Mat. Sci. Tech. 7, 427-439. 